\title{
Lorentz distributed noncommutative wormhole solutions in extended teleparallel gravity
}

\author{
Abdul Jawad ${ }^{\mathrm{a}}$, Shamaila Rani ${ }^{\mathrm{b}}$ \\ Department of Mathematics, COMSATS Institute of Information Technology, Lahore, Pakistan
}

Received: 19 January 2015 / Accepted: 2 April 2015 / Published online: 28 April 2015

(C) The Author(s) 2015. This article is published with open access at Springerlink.com

\begin{abstract}
In this paper, we study static spherically symmetric wormhole solutions in extended teleparallel gravity with the inclusion of noncommutative geometry under a Lorentzian distribution. We obtain expressions of matter components for a non-diagonal tetrad. The effective energymomentum tensor leads to the violation of energy conditions which impose a condition on the normal matter to satisfy these conditions. We explore the noncommutative wormhole solutions by assuming a viable power-law $f(T)$ and shape function models. For the first model, we discuss two cases in which one leads to teleparallel gravity and the other is for $f(T)$ gravity. The normal matter violates the weak energy condition for the first case, while there exists a possibility for micro physically acceptable wormhole solution. There exists a physically acceptable wormhole solution for the power-law $b(r)$ model. Also, we check the equilibrium condition for these solutions, which is only satisfied for the teleparallel case, while for the $f(T)$ case, these solutions are less stable.
\end{abstract}

\section{Introduction}

General theory of relativity laid down the foundation of modern cosmology. The $\Lambda \mathrm{CDM}$ model is the simplest model compatible with all cosmological observations but suffers from some shortcomings, like the fine-tuning and cosmic coincidence problems. The modified theories of gravity are generalized models that came into being by modifying the gravitational part in the general relativity (GR) action, while the matter part remains unchanged. At large distances, these theories modify the dynamics of the universe. In another scenario, the modified matter part with unchanged gravitational part results in dynamical models, such as cosmological constant, quintessence, $k$-essence, Chaplygin gas, and

\footnotetext{
ae-mails: jawadab181@yahoo.com; abduljawad@ciitlahore.edu.pk

be-mails: drshamailarani@ciitlahore.edu.pk; shamailatoor.math@yahoo.com
}

HDE models [1-5]. After GR, Einstein attempted to unify gravitation and electromagnetism based on the mathematical structure of absolute or distant parallelism, also referred to as teleparallelism, which led to teleparallel (TP) gravity. In this gravity, the gravitational field is established through torsion using the Witzenböck connection instead of curvature via the Levi-Civita connection as in GR.

The extended TP theory of gravity [or $f(T)$ gravity where $T$ represents the torsion scalar] is the generalization of TP gravity in the same fashion as $f(R)$ gravity generalizes GR. This is attained by replacing the torsion scalar in the action of TP gravity by a general differentiable function $f(T)$. Ferraro and Fiorini $[6,7]$ firstly introduced this theory by applying a Born-Infeld strategy and solved the particle horizon problem as well as obtained singularity-free solutions with a positive cosmological constant. Afterwards, this theory has been studied extensively as regards many phenomena, like the accelerated expansion of the universe, solar system constraints, a discussion of Birkhoff's theorem, thermodynamics, a reconstruction via dynamical models, static and dynamical wormhole solutions, the viability of models through a cosmographic technique, instability ranges of collapsing stars, and many more. A vast area of research in $f(T)$ gravity uses a spherically symmetric scenario [8-22].

A wormhole is a hypothetical path to connect different regions of the universe, which can be regarded as a tunnel or bridge from which an observer may traverse easily. Wormhole solutions are described by static as well as dynamical configurations. In GR, the exotic matter (i.e., violating the energy condition) constitutes a basic ingredient in the development of the mathematical structure of the wormhole. The violation of NEC is the necessary tool to form wormhole solutions which also allow for two-way travel. Also, the inclusion of an electromagnetic field, a scalar field, noncommutative (NC) geometry, a NC Lorentzian (NCL) distribution, [23-26] etc. establish more interesting and useful results. The search for a realistic source which provides the violation (while 
normal matter satisfies the energy conditions) has recently gained a lot of interest. The modified theory of gravity is one of the directions to establish realistic wormhole solutions.

In $f(T)$ gravity, the effective energy-momentum tensor is the cause for the corresponding violation, while normal matter threads the wormhole solutions. In this regard, the extra terms related to the underlying theory play an effective role in violating the energy conditions, which is necessary to keep wormhole throat open to be traversable. Böhmerbreak et al. [27] were the first who studied wormhole solutions in this gravity by taking static spherically symmetric traversable wormhole solutions and found some constraints on the wormhole throat. They explored the behavior of the energy conditions by taking a particular $f(T)$, shape, and redshift functions and obtained physically acceptable solutions. Jamilbreak et al. [28] studied these solutions by taking the fluid isotropic and anisotropic, as well as choosing a particular equation of state; they found that the energy conditions are violated in the anisotropic case, while these are satisfied for the remaining two cases. Sharif and Rani [29] explored dynamical wormhole solutions for traceless as well as barotropic equations of state. With the help of analytic and numerical $f(T)$ models, they concluded that the weak energy condition (WEC) holds in specific time intervals for these cases.

Rahaman et al. [30] examined the NC wormhole solutions in GR for a higher dimensional static spherically symmetric spacetime and found their existence in a regular way up to four dimensions, and in a very restrictive way for fivedimensional space. Beyond these dimensions, the possibility of wormhole solutions is over. Jamil et al. [31] also explored $f(R)$ wormhole solutions in the same background. Recently, Sharif and Rani have studied wormhole solutions [32] in the background of NC geometry for $f(T)=\alpha T^{n}$ model as well as the shape function. They concluded that the effective energy-momentum tensor serves as the basic ingredient to thread the wormhole solutions, and normal matter gives some physically acceptable solutions. They extended [33] this work to a study of the effects of an electrostatic field. The same authors explored these solutions for galactic halo regions [34] for exponential and logarithmic models but no physically acceptable solutions are obtained.

Recently, Bhar and Rahaman [35] studied the wormhole solutions in Lorentzian distribution as the density function in a noncommutativity-inspired spacetime. They obtained a stable wormhole which is asymptotically flat in the usual four-dimensional spacetime. In order to search for some realistic wormhole solutions, we extended this work in extended teleparallel gravity for specific $f(T)$ and shape function models. The paper is organized as follows. In the next section, we provide wormhole geometry for a static spherically symmetric spacetime and briefly discuss the energy conditions. Section 3 is devoted to a discussion of $f(T)$ gravity. In Sect.
4, we construct the field equations and matter content for the wormhole solutions. Section 5 contains the construction of wormhole solutions for particular $f(T)$ and $b(r)$ functions. In Sect. 6, we check the equilibrium condition for the obtained solutions. The last section summarizes the results.

\section{Wormhole geometry}

One of the most fascinating features of GR is the possible existence of spacetimes with a non-trivial topological structure. Well-known examples of this structure are described by Misner and Wheeler [36] and Wheeler [37] as solutions of the Einstein field equations known as wormhole solutions. Basically, the wormhole, having appearance as a tube, a tunnel or a bridge, represents a shortcut way to communicate between two distant regions. If there exist some other paths between these regions, then these are called "intra-universe", otherwise "inter-universe" wormholes. The simplest example of such a connection is the Einstein-Rosen bridge (or Schwarzschild wormhole), which unfortunately fails to provide a way of communication to another region of space to which it is connected. The reason behind this is that any photon or particle falling in it reaches the singularity at $r=0$ and thus has no link with the other end of the wormhole.

The Lorentzian traversable wormholes are the most favorable in the sense that a human may traverse from one side of the wormhole to the other through these wormholes. Being the generalized form of the Schwarzschild wormhole (having an event horizon which permits one way travel) with no event horizon, these wormholes lead to two-way travel. Morris and Thorne [38] established a static spherically symmetric wormhole spacetime given by

$\mathrm{d} s^{2}=e^{2 \Psi(r)} \mathrm{d} t^{2}-\frac{\mathrm{d} r^{2}}{1-\frac{b(r)}{r}}-r^{2} \mathrm{~d} \theta^{2}-r^{2} \sin ^{2} \theta \mathrm{d} \phi^{2}$.

Here, $\Psi(r)$ represents the redshift (or potential) function which determines the gravitational redshift of a light particle (photon). The magnitude of the redshift function must be finite everywhere for the two-way travel through the wormhole. The function $b(r)$ denotes the shape function which sets the shape of the wormhole.

The essential characteristics required for a wormhole geometry are discussed in $[38,39]$ and given as follows.

- The no-horizon condition [ $\Psi(r)$ must be finite everywhere in the spacetime] must be satisfied by the redshift function. Usually, it is taken as zero, which gives $e^{2 \Psi(r)} \rightarrow 1$.

- The shape function must satisfy the flaring out condition on the throat, i.e., to have the proper shape for a wormhole, the ratio of the radial coordinate to the shape function at that coordinate must be 1 , while this coordinate expresses 
a non-monotonic behavior away from the throat. Taking the throat radius as $r_{0}$ yields $b\left(r_{0}\right)=r_{0}$ and $b^{\prime}\left(r_{0}\right)<1$.

- The proper radial distance, $\tau(r)= \pm \int_{r_{0}}^{r} \frac{\mathrm{d} r}{\sqrt{1-b(r) / r}}$, $r \geq r_{0}$, should be finite throughout the space. The signs \pm associate the two parts which are joined by the throat for the wormhole configuration.

- At large distances, the asymptotic flatness should be a feature of the spacetime, i.e., $\frac{b(r)}{r} \rightarrow 0$ as $r \rightarrow \infty$.

Notice that the Morris-Thorne wormhole is not a particular wormhole solution like the Schwarzschild wormhole, which depends on a single parameter, the mass of the wormhole. Instead, it is a class of solutions for an arbitrarily large number of redshift as well as shape functions satisfying the above constraints.

In order to prevent shrinking of the wormhole throat and to make it traversable, the matter distribution of the energymomentum tensor at the throat must be negative. More precisely, the sum of the energy density and the pressure of matter is negative, representing a violation of the null energy condition (NEC) and such matter is named "exotic". It is noted that an ordinary energy-momentum tensor satisfies the NEC. For viability of the wormhole solutions, it is necessary to minimize the amount of exotic matter required to support the wormhole solutions. The modified theories of gravity are one of the sources which provide an effective energy-momentum tensor to violate the WEC (contains NEC). In this regard, the usual energy-momentum tensor may satisfy this condition. To discuss NEC and WEC, we assume an energy-momentum tensor in an appropriate orthonormal frame as

$\mathcal{T}^{\alpha \beta}=\operatorname{diag}\left(\rho, p_{1}, p_{2}, p_{3}\right)$

where $\rho$ is the energy density and $p_{n}(n=1,2,3)$ denote the principal pressures.

- WEC The relationship between the Raychaudhuri equation and attractiveness of gravity yields the WEC

$$
\mathcal{T}_{\alpha \beta} V^{\alpha} V^{\beta} \geq 0
$$

for any timelike vector $V^{\alpha}$. In terms of the components of the energy-momentum tensor, this inequality yields

$$
\rho \geq 0, \quad \rho+p_{n} \geq 0, \quad \forall n
$$

For modified theories of gravity, we replace the matter content of the universe in an effective manner by $\mathcal{T}_{\alpha \beta}^{\text {eff }}$ as well as the matter components $\rho^{\text {eff }}$ and $p_{n}^{\text {eff }}$.

- NEC By continuity, the WEC implies the NEC,

$$
\mathcal{T}_{\alpha \beta} k^{\alpha} k^{\beta} \geq 0
$$

for any null vector $k^{\alpha}$. This inequality gives $\rho+p_{n} \geq 0, \forall n$. In an effective manner, this becomes $\rho^{\text {eff }}+p_{n}^{\text {eff }} \geq 0$.

\section{$3 f(T)$ theory of gravity}

It is well known that curvature and torsion are properties of a connection and many connections may be defined on the same spacetime. The Riemann-Cartan (generalized Riemannian) spacetime yields two interesting models of spacetime such as Riemannian and Weitzenböck spacetimes [40,41]. The concept of a curved manifold is a crucial characteristic of GR which is described by a Riemannian spacetime having the metric tensor as the dynamical variable. It uses a curvature (Riemann) tensor to arrive at a Levi-Civita connection whose curvature remains non-zero while the torsion vanishes due to its symmetry property. On the other hand, the non-zero torsion with vanishing curvature corresponds to the Weitzenböck spacetime which parallel transports the tetrad field. The TP theory is defined by a Weitzenböck connection with a tetrad field as the basic entity. This theory is an alternative description of gravity having a translation group which is related to a gauge theory. The existence of nontrivial tetrad field in gauge theory leads to the teleparallelism structure. The $f(T)$ theory of gravity is the generalization of TP theory.

The geometry of TP theory is uniquely defined by an orthonormal set of four-vector fields (three spacelike and one timelike) named the tetrad field. The simplest type of tetrad field is the trivial tetrad, which has the form $e_{i}=\delta_{i}^{\mu} \partial_{\mu}, e^{j}=$ $\delta_{\mu}^{j} \mathrm{~d} x^{\mu}$, where $\delta_{\mu}^{i}$ is the Kronecker delta. However, this type of tetrad field gives a zero torsion and is of less interest. The non-trivial tetrad field provides non-zero torsion and yields the construction of TP theory. It can be written as

$h_{i}=h_{i}{ }^{\mu} \partial_{\mu}, \quad h^{j}=h^{j}{ }_{\nu} \mathrm{d} x^{\nu}$

satisfying the following properties: $h^{i}{ }_{\mu} h_{j}{ }^{\mu}=\delta_{j}^{i}, h^{i}{ }_{\mu} h_{i}{ }^{\nu}=$ $\delta_{\mu}^{v}$. This field establishes the metric tensor as a by-product given as follows:

$g_{\mu \nu}=\eta_{i j} h_{\mu}^{i} h_{\nu}^{j}$.

The torsion scalar has the following form:

$T=S_{\gamma}^{\mu \nu} T_{\mu \nu}^{\gamma}$

The superpotential tensor $S_{\gamma}^{\mu \nu}$ (antisymmetric in its upper indices) is

$S_{\gamma}^{\mu \nu}=\frac{1}{2}\left(K^{\mu \nu}{ }_{\gamma}+\delta_{\gamma}^{\mu} T_{\theta}^{\theta v}-\delta_{\gamma}^{\nu} T_{\theta}^{\theta \mu}\right)$. 
The torsion tensor is as follows:

$T_{\mu \nu}^{\gamma}=\widetilde{\Gamma}_{\nu \mu}^{\gamma}-\widetilde{\Gamma}_{\mu \nu}^{\gamma}=h_{i}{ }^{\gamma}\left(\partial_{\nu} h_{\mu}^{i}-\partial_{\mu} h^{i}{ }_{\nu}\right)$,

which is antisymmetric in its lower indices, i.e., $T^{\gamma}{ }_{\mu \nu}=$ $-T^{\gamma}{ }_{\nu \mu}$. The contorsion tensor can be defined as

$K_{\mu \nu}^{\gamma}=\frac{1}{2}\left[T_{\mu}{ }_{\nu}+T_{\nu}{ }_{\mu}-T_{\mu \nu}^{\gamma}\right]$.

To formulate a suitable form of the field equations which establishes the equivalent description (up to equations level) of these theories, we follow the covariant formalism [42-45]. Incorporating the above equations and after some algebraic manipulations, it follows that

$G_{\mu \nu}-\frac{1}{2} g_{\mu \nu} T=-\nabla^{\gamma} S_{\nu \gamma \mu}-S^{\sigma \gamma}{ }_{\mu} K_{\gamma \sigma \nu}$,

where $G_{\mu \nu}=R_{\mu \nu}-\frac{1}{2} g_{\mu \nu} R$ is the Einstein tensor. Finally, we obtain the following field equations for $f(T)$ gravity:

$f_{T} G_{\mu \nu}+\frac{1}{2} g_{\mu \nu}\left(f-T f_{T}\right)+E_{\mu \nu} f_{T T}=\kappa^{2} \mathcal{T}_{\mu \nu}$,

where $E_{\mu \nu}=S_{\nu \mu}{ }^{\gamma} \nabla_{\gamma} T$. It is noted that Eq. (9) possesses an equivalent structure like $f(R)$ gravity and reduces to GR for $f(T)=T$. The trace equation is used to constrain and simplify the field equations. Here, the trace of the above equation is

$E f_{T T}-(R+2 T) f_{T}+2 f=\kappa^{2} \mathcal{T}$

with $E=E^{v}{ }_{v}$ and $\mathcal{T}=\mathcal{T}^{v}{ }{ }_{v}$. In terms of an effective energymomentum tensor, the $f(T)$ field equations can be rewritten as

$G_{\mu \nu}=\kappa^{2} \mathcal{T}_{\mu \nu}^{\text {eff }}=\kappa^{2}\left(\mathcal{T}_{\mu \nu}^{f}+\mathcal{T}_{\mu \nu}^{T}\right)$.

The term $\mathcal{T}_{\mu \nu}^{f}=\frac{\mathcal{T}_{\mu \nu}^{m}}{f_{T}}$ corresponds to the matter fluid, while, using the trace equation, the torsion contribution is given by

$\mathcal{T}_{\mu \nu}^{T}=\frac{1}{\kappa^{2} f_{T}}\left[-E_{\mu \nu} f_{T T}-\frac{1}{4} g_{\mu \nu}\left(\mathcal{T}-E f_{T T}+R f_{T}\right)\right]$.

\section{Field equations for wormhole construction}

Gravitational theories [like $f(R)$ theory] developed through the metric tensor as well as its dependent quantities are local Lorentz invariant. Being the basic entity in $f(T)$ gravity, the torsion tensor (taking a tetrad) which induces the TP structure on spacetime fails to be invariant under local Lorentz transformations [42-45]. This can be seen from
$R=-T-2 \nabla^{\gamma} T^{\nu}{ }_{\gamma \nu}$, where $R$ is a covariant scalar, while $T$ as well as $\nabla^{\gamma} T^{v}{ }_{\gamma \nu}$ are covariant scalars but not local Lorentz invariant. However, the latter scalar can be neglected inside the integral for TP theory and it becomes equivalent to GR.

The non-invariant theories might be sensitive in order to choose a reasonable tetrad not uniquely determined by the given metric $g_{\mu \nu}$. In general, one comes across a more complicated connection between the tetrad and metric, particularly for a non-diagonal tetrad with a diagonal metric (or even sometimes a diagonal tetrad). Different field equations are developed by taking a different tetrad, which successively induces distinct solutions. In an appropriate limit, some of these solutions lead to GR counterparts, while others fail to provide a valid GR counterpart. Thus, the choice of tetrad is a crucial point in $f(T)$ theory and needs particular attention.

When we deal with spherical coordinates, the diagonal tetrads become unsuitable as they provide some constraints on the $T$ and $f(T)$ models [46]. We obtain an unwanted condition, $\dot{T} f_{T T}=0$ (or simply $f_{T T}=0$ ), which yields a constant torsion scalar, or $f(T)=c_{1}+c_{2} T$ representing TP theory. Thus, the diagonal tetrads do not represent a useful choice for spherical symmetry. In order to search for a realistic source toward wormhole solutions in $f(T)$ gravity, we assume the following non-diagonal tetrad for static spherically symmetric wormhole spacetime (1):

$$
\begin{aligned}
& h^{i} \mu \\
& =\left(\begin{array}{cccc}
e^{2 \Psi(r)} & 0 & 0 & 0 \\
0 & \frac{1}{\sqrt{1-\frac{b}{r}}} \sin \theta \cos \phi & r \cos \theta \cos \phi & -r \sin \theta \sin \phi \\
0 & \frac{1}{\sqrt{1-\frac{b}{r}}} \sin \theta \sin \phi & r \cos \theta \sin \phi & r \sin \theta \cos \phi \\
0 & \frac{1}{\sqrt{1-\frac{b}{r}}} \cos \theta & -r \sin \theta & 0
\end{array}\right) .
\end{aligned}
$$

The torsion scalar turns out to be

$$
\begin{aligned}
T= & -\frac{2}{r}\left[2 \Psi^{\prime}\left(\sqrt{1-\frac{b}{r}}-1+\frac{b}{r}\right)\right. \\
& \left.-\frac{1}{r}\left(2\left(1-\sqrt{1-\frac{b}{r}}\right)-\frac{b}{r}\right)\right] .
\end{aligned}
$$

We assume an anisotropic energy-momentum tensor as

$$
\mathcal{T}_{v}^{\mu}=\left(\rho+p_{t}\right) U^{\mu} U_{v}-p_{t} \delta_{v}^{\mu}+\left(p_{r}-p_{t}\right) V^{\mu} V_{v},
$$

where $p_{r}$ and $p_{t}$ are the radial and transverse components of the pressure. The four-velocity of the fluid $U^{\mu}$ and the unit spacelike vector $V^{\mu}$ satisfy $U^{\mu} U_{\mu}=1, V^{\mu} V_{\mu}=$ $-1, U^{\mu} V_{\mu}=0$. The corresponding energy-momentum tensor is

$\mathcal{T}^{\mu}=\operatorname{diag}\left(\rho,-p_{r},-p_{t},-p_{t}\right)$. 
Equation (9) yields the following field equations:

$$
\begin{aligned}
& \frac{\rho}{f_{T}}-\frac{1}{r}\left(1-\frac{b}{r}-\sqrt{1-\frac{b}{r}}\right) T^{\prime} \frac{f_{T T}}{f_{T}}-\frac{J}{f_{T}}=\frac{b^{\prime}}{r^{2}} \\
& \frac{p_{r}}{f_{T}}+\frac{J}{f_{T}}=\frac{1}{r^{2}}\left[2 r \Psi^{\prime}\left(1-\frac{b}{r}\right)-\frac{b}{r}\right] \\
& \frac{p_{t}}{f_{T}}+\frac{1}{2}\left\{\Psi^{\prime}\left(1-\frac{b}{r}\right)-\frac{1}{r}\left(\sqrt{1-\frac{b}{r}}-1+\frac{b}{r}\right)\right\} \\
& \quad \times T^{\prime} \frac{f_{T T}}{f_{T}}+\frac{J}{f_{T}}=\frac{1}{2 r^{2}}\left[2 r \Psi^{\prime}-\Psi^{\prime} b-b^{\prime}+\frac{b}{r}+2 \Psi^{\prime \prime} r^{2}\right. \\
& \left.\quad-2 \Psi^{\prime \prime} r b+2 \Psi^{\prime 2} r^{2}-2 \Psi^{\prime 2} r b-\Psi^{\prime} r b^{\prime}\right]
\end{aligned}
$$

where the prime refers to the derivative with respect to $r$ and $J(r)$ is given by

$$
J(r)=\frac{1}{4}\left(\mathcal{T}-E f_{T T}+R f_{T}\right)
$$

Taking the effective energy density and the pressure from Eqs. (14) and (15), the NEC yields

$\rho^{\mathrm{eff}}+p_{r}^{\mathrm{eff}}=\frac{b^{\prime} r-b}{r^{3}}+\frac{2}{r}\left(1-\frac{b}{r}\right) \Psi^{\prime}$.

Due to the flaring out condition, we obtain $b>b^{\prime} r$, leading to the violation of the NEC with $\Psi^{\prime}<0$, i.e., $\rho^{\text {eff }}+p_{r}^{\text {eff }} \leq 0$, which implies that the effective energy-momentum tensor is responsible for the necessary violation of the energy conditions to support the wormhole geometry. Thus, it may impose a condition on the usual matter to satisfy the energy conditions in this scenario and establish some physically acceptable solutions.

To be a traversable wormhole solution, the magnitude of its redshift function must be finite. Also, up to equation level for a constant value of $\Psi$ other than zero, we note that only $\Psi^{\prime}$ is used for which $\Psi=$ constant gives the same scenario. For the sake of simplicity, setting $\Psi=0$ in Eqs. (14)-(16), the field equations can be written as

$$
\begin{aligned}
\rho & =\frac{b^{\prime}}{r^{2}} f_{T}+\frac{1}{r}\left(1-\frac{b}{r}-\sqrt{1-\frac{b}{r}}\right) T^{\prime} f_{T T}, \\
p_{r} & =-\frac{b}{r^{3}} f_{T} \\
p_{t} & =\frac{1}{2 r}\left(\sqrt{1-\frac{b}{r}}-1+\frac{b}{r}\right) T^{\prime} f_{T T}-\frac{1}{2 r^{2}}\left(b^{\prime}-\frac{b}{r}\right) f_{T},
\end{aligned}
$$

where the torsion scalar becomes

$$
T=\frac{2}{r^{2}}\left[2-\frac{b}{r}-2 \sqrt{1-\frac{b}{r}}\right]
$$

It is noted that Eq. (17) gives the violation of the NEC in terms of the flaring out condition.

\section{Wormhole solutions}

Noncommutative geometry is the intrinsic characteristic of spacetime and it plays an effective role in several areas. To formulate the NC form of GR, the coordinate coherent state approach is widely used. The NC geometry is used to eliminate the divergencies that appear in GR by replacing the point-like structures with smeared objects. Taking a Lorentzian distribution, the energy density of the particlelike static spherically symmetric gravitational source having mass $M$ takes the following form [47]:

$\rho_{\mathrm{NCL}}=\frac{M \sqrt{\phi}}{\pi^{2}\left(r^{2}+\phi\right)^{2}}$,

where $\phi$ is the NC parameter. Taking into account the correspondence between $\rho$ and $\rho_{\mathrm{NCL}}$ using Eqs. (18) and (22), we obtain the following differential equation:

$\frac{b^{\prime}}{r^{2}} f_{T}+\frac{1}{r}\left(1-\frac{b}{r}-\sqrt{1-\frac{b}{r}}\right) T^{\prime} f_{T T}=\frac{M \sqrt{\phi}}{\pi^{2}\left(r^{2}+\phi\right)^{2}}$,

which contains two unknown functions, $b$ and $f$. Thus, we have to choose one of these functions and carried out steps for the other one. We discuss the NCL wormhole solutions in $f(T)$ gravity for a non-diagonal tetrad and investigate the behavior of the energy conditions for specific $f(T)$ and shape functions.

\subsection{For power-law $f(T)$ model}

We assume a model in power-law form of the torsion scalar which is the generalization of GR and an analogy to $f(R)$ model like $f(R)=R+\delta R^{2}$ taken to discuss the wormhole solutions. The $f(T)$ model is

$f(T)=T+\alpha T^{2}$.

This model has contributed as the most viable model due to its simple form and we may directly compare our results with GR. We discuss wormhole solutions for the following two cases.

Case I: $\alpha=0$

We consider $\alpha=0$ in model (24) which leads to the whole scenario in teleparallel gravity. In this case, Eq. (23) becomes

$\frac{b^{\prime}}{r^{2}}=\frac{M \sqrt{\phi}}{\pi^{2}\left(r^{2}+\phi\right)^{2}}$, 
(i)

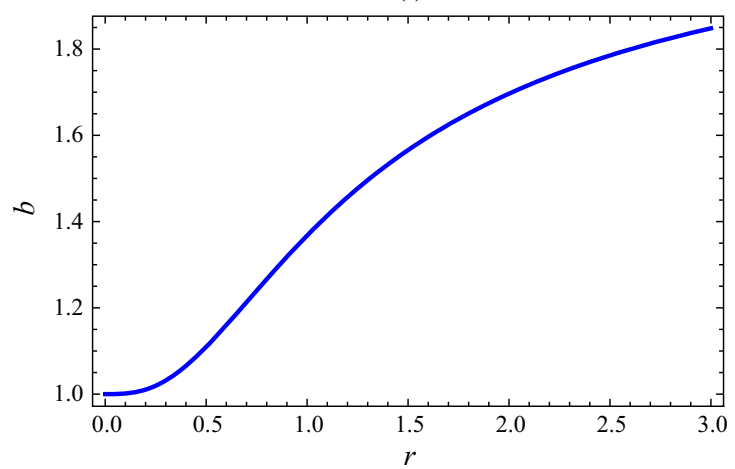

(iii)

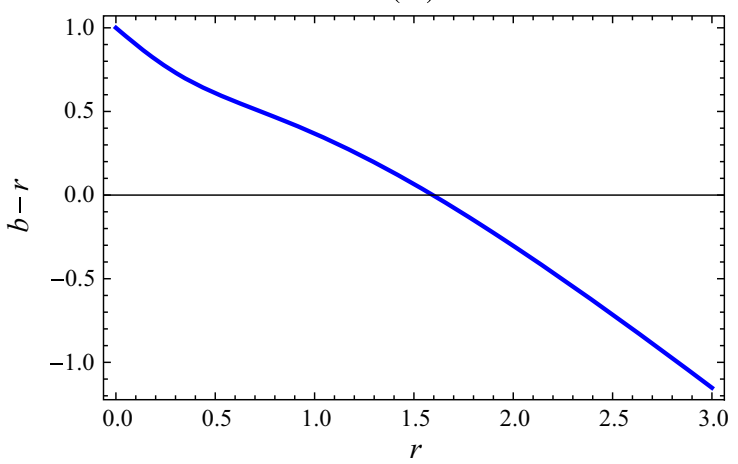

(ii)

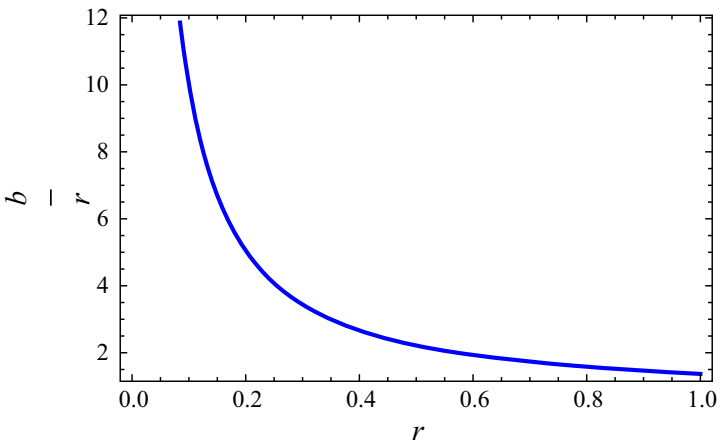

(iv)

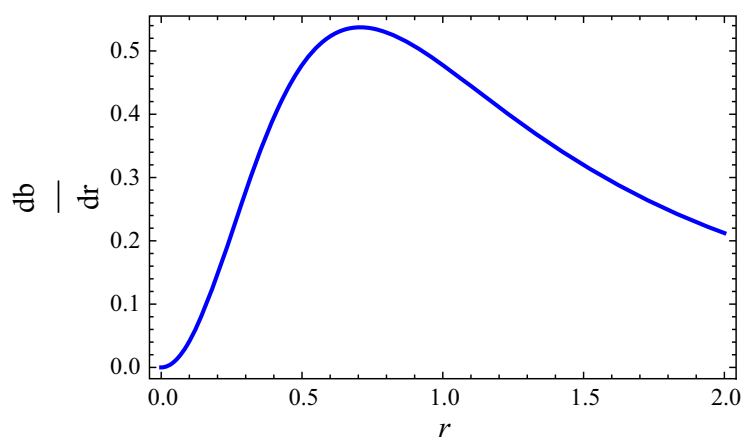

Fig. 1 Plots of shape function in the teleparallel case: evolution of $\mathbf{i} b$ versus $r$, ii $\frac{b}{r}$ versus $r$, iii $b-r$ versus $r$, iv $\frac{\mathrm{d} b}{\mathrm{~d} r}$ versus $r$

yielding the solution

$b(r)=\frac{M}{2 \pi^{2}}\left(\frac{r \sqrt{\phi}}{r^{2}+\phi}+\tan ^{-1}\left(\frac{r}{\sqrt{\phi}}\right)\right)+c$,

where $c$ is an arbitrary constant.

In order to examine the geometry of the wormhole, we draw the shape function taking different conditions in Fig. 1. We choose arbitrarily the values of the model parameters, such as $\phi=0.5, M=15$, and $c=1$. Figure 1 i represents the evolution of the shape function in an increasing manner versus $r$. For an asymptomatically flat condition, we draw $\frac{b}{r}$ with respect to increasing $r$. It can be seen from plot ii that $\frac{b}{r}$ approaches 0 as $r \rightarrow 0$. This implies that the asymptotically flat condition for the wormhole construction is satisfied. In plot iii, we draw $b-r$ versus $r$ to find the throat radius. It is noted that the throat radius is the minimum value for which $b-r$ cuts the $r$-axis. In this case, the throat radius is obtained as $r_{0}=1.6$. This plot satisfies the condition $1-\frac{b}{r}>0$ for $r>r_{0}$. In Fig. 1iv, we plot the first derivative of $b(r)$ with respect to $r$ to check the validity of the condition $b^{\prime}\left(r_{0}\right)<1$, which shows that the corresponding condition is satisfied. Thus, the shape function satisfies the required structure of the wormhole.
Equations (18)-(20) are given as follows:

$$
\begin{aligned}
\rho= & \frac{M \sqrt{\phi}}{\pi^{2}\left(r^{2}+\phi\right)^{2}}, \\
p_{r}= & -\frac{1}{r^{3}}\left[\frac{M}{2 \pi^{2}}\left(\frac{r \sqrt{\phi}}{r^{2}+\phi}+\tan ^{-1}\left(\frac{r}{\sqrt{\phi}}\right)\right)+c\right], \\
p_{t}= & -\frac{1}{2 r^{2}}\left[\frac{M \sqrt{\phi}}{\pi^{2}\left(r^{2}+\phi\right)^{2}}\right. \\
& \left.-\frac{1}{r}\left\{\frac{M}{2 \pi^{2}}\left(\frac{r \sqrt{\phi}}{r^{2}+\phi}+\tan ^{-1}\left(\frac{r}{\sqrt{\phi}}\right)\right)+c\right\}\right] .
\end{aligned}
$$

The behavior of WEC $\left(\rho, \rho+p_{r}\right.$, and $\left.\rho+p_{t}\right)$ versus $r$ is shown in Fig. 2. The curves of $\rho, \rho+p_{t}$ represent a positively decreasing behavior for increasing $r$, while $\rho+p_{r}$ indicates a negative behavior, which shows the violation of WEC. Thus no physically acceptable wormhole solution is obtained in the teleparallel case. These results are compatible with the results of [35].

Case II: $\alpha \neq 0$

The case $\alpha \neq 0$ leads to the extended teleparallel gravity. Inserting Eq. (24) in Eq. (23), we obtain the following differential equation: 
(i)

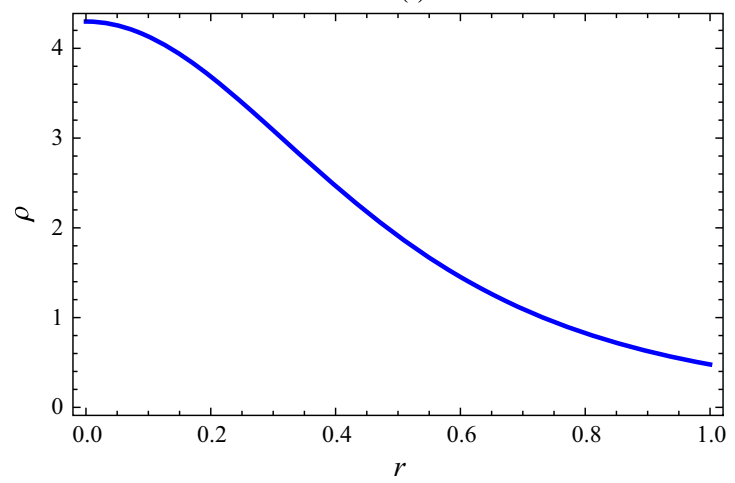

(ii)

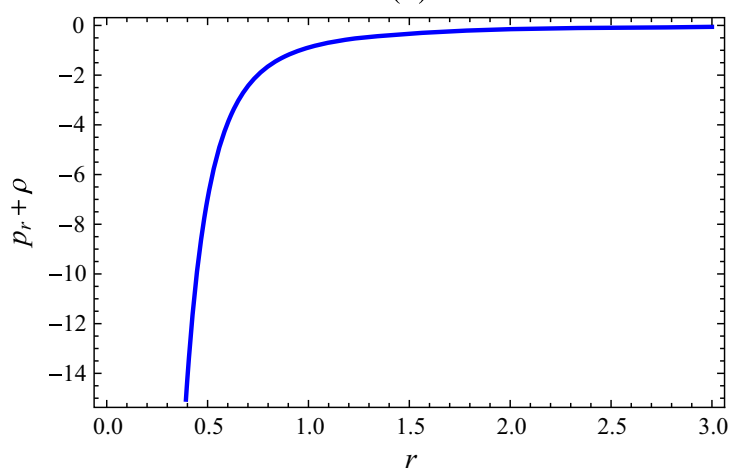

(iii)

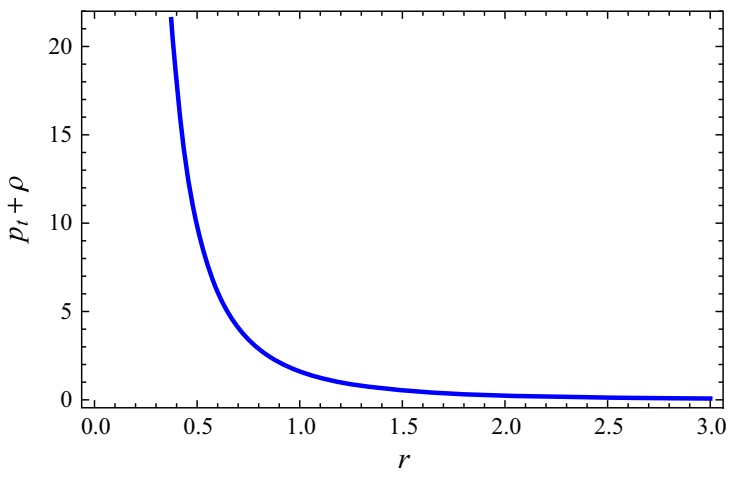

Fig. 2 Plots of WEC in teleparallel case: $\mathbf{i} \rho$ versus $r$, ii $\rho+p_{r}$ versus $r$, iii $\rho+p_{t}$ versus $r$

$$
\begin{aligned}
\frac{r^{2} M \sqrt{\phi}}{\pi^{2}\left(r^{2}+\phi\right)^{2}}= & b^{\prime}\left\{1+\frac{4 \alpha}{r^{2}}\left(2-\frac{b}{r}-2 \sqrt{1-\frac{b}{r}}\right)\right\} \\
& -\frac{4 \alpha}{r^{2}}\left(1-\frac{b}{r}-\sqrt{1-\frac{b}{r}}\right) \\
& \times\left[4\left(1-\sqrt{1-\frac{b}{r}}\right)-\frac{2 b}{r}\right. \\
& \left.+\frac{b^{\prime} r-b}{r}\left(1-\frac{1}{\sqrt{1-\frac{b}{r}}}\right)\right] .
\end{aligned}
$$

We check the behavior of the shape function and the flaring out conditions numerically through the graphs by taking the same values of parameters along with three different values of $\alpha$ such as $\alpha=-2,-3,-5$ and the initial value as $f(2.2)=$ 1. Figure $3 i$ represents an increasing behavior of the shape function versus $r$. In the right graph, ii, $\frac{b}{r}$ versus $r$ shows that $\frac{b}{r}$ approaches zero as we increase $r$, which represents that asymptomatically flatness is obtained. To locate the throat of the wormhole, we plot $b(r)-r$ versus $r$ as shown in plot iii of Fig. 3. In this plot, the throat is located at very small values of $r$. The first derivative of the shape function is also plotted versus $r$ as shown in plot iv, which indicates that $\frac{\mathrm{d} b}{\mathrm{~d} r}$ at $r_{0}$ satisfies the condition $b^{\prime}\left(r_{0}\right)<1$. Thus, similar to the teleparallel case, the shape function satisfies the conditions of wormhole geometry.

To check the behavior of WEC for power-law model, the expressions of matter content using Eqs. (18)-(20) are given by

$$
\begin{aligned}
\rho= & \frac{b^{\prime}}{r^{2}}\left\{1+\frac{4 \alpha}{r^{2}}\left(2-\frac{b}{r}-2 \sqrt{1-\frac{b}{r}}\right)\right\} \\
& -\frac{4 \alpha}{r^{2}}\left(1-\frac{b}{r}-\sqrt{1-\frac{b}{r}}\right)\left[4\left(1-\sqrt{1-\frac{b}{r}}\right)-\frac{2 b}{r}\right. \\
& \left.+\frac{b^{\prime} r-b}{r}\left(1-\frac{1}{\sqrt{1-\frac{b}{r}}}\right)\right], \\
p_{r}= & -\frac{b}{r^{3}}\left\{1+\frac{4 \alpha}{r^{2}}\left(2-\frac{b}{r}-2 \sqrt{1-\frac{b}{r}}\right)\right\}, \\
p_{t}= & -\frac{1}{2 r^{2}}\left(b^{\prime}-\frac{b}{r}\right)\left\{1+\frac{4 \alpha}{r^{2}}\left(2-\frac{b}{r}-2 \sqrt{1-\frac{b}{r}}\right)\right\} \\
& -\frac{2 \alpha}{r^{4}}\left(1-\frac{b}{r}-\sqrt{1-\frac{b}{r}}\right)\left[4\left(1-\sqrt{1-\frac{b}{r}}\right)-\frac{2 b}{r}\right. \\
& \left.+\frac{b^{\prime} r-b}{r}\left(1-\frac{1}{\sqrt{1-\frac{b}{r}}}\right)\right] .
\end{aligned}
$$


(i)

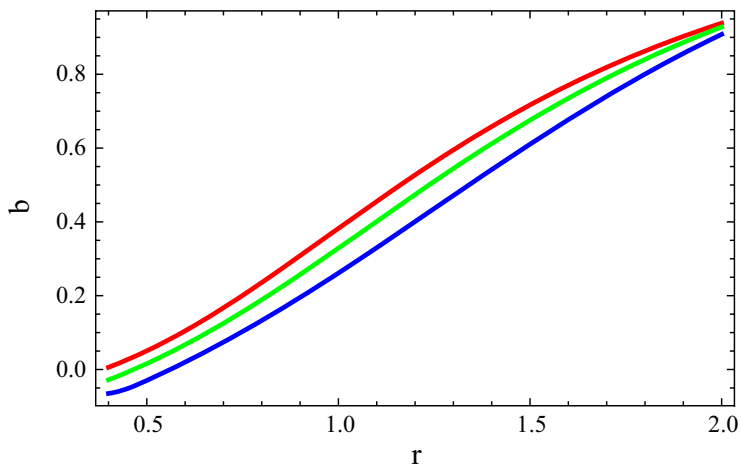

(iii)

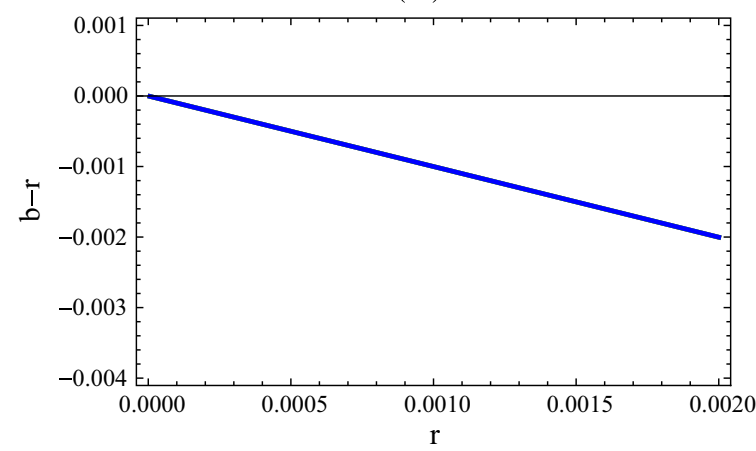

(ii)

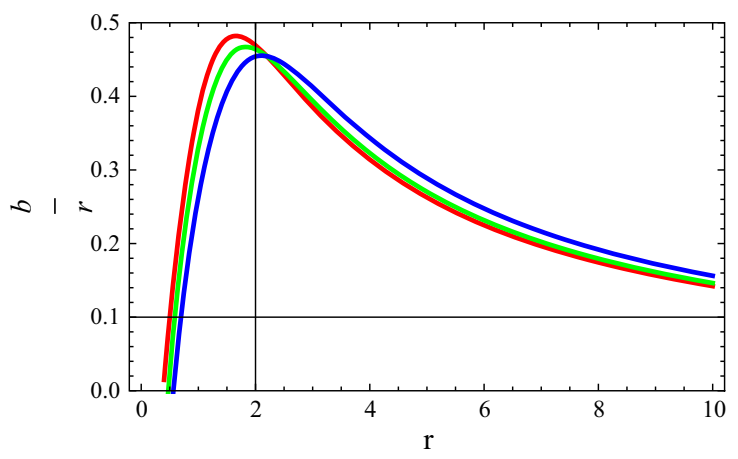

(iv)

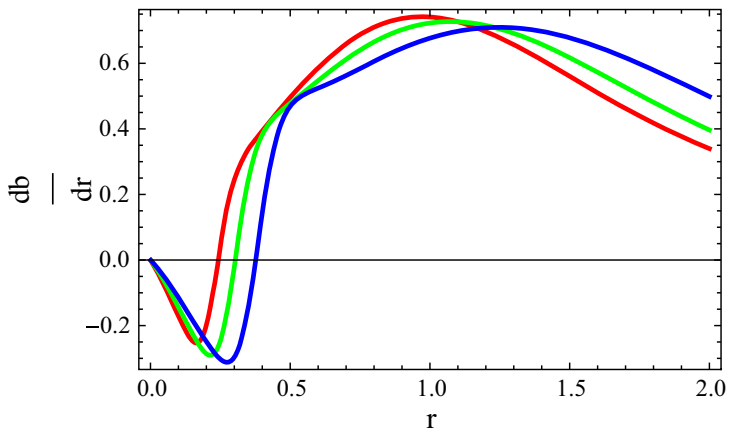

Fig. 3 Plots of shape function in $f(T)$ case: red curve for $\alpha=-2$, green curve for $\alpha=-3$, blue curve for $\alpha=-5$. Evolution of $\mathbf{i} b$ versus $r$, ii $\frac{b}{r}$ versus $r$, iii $b-r$ versus $r$, iv $\frac{\mathrm{d} b}{\mathrm{~d} r}$ versus $r$

Figure 4 represents a graph of the WEC expressions versus $r$, which show that $\rho$ and $\rho+p_{t}$ show a decreasing behavior but remain positive. For $\alpha=-2, \rho$ indicates a negative value at $r \leq 0.52$. The behavior of $\rho+p_{r}$ is negative but there exists some part of the curves in the positive panel of the plot. Thus there exists a possibility to have a micro or tiny wormhole.

\subsection{For power-law $b(r)$ model}

Here, we examine the wormhole solution by considering a specific shape function in terms of the power-law form and construct the $f(T)$ function in the NCL background. We take the following particular shape function $[31,50,51]$ :

$b(r)=r_{0}\left(\frac{r}{r_{0}}\right)^{\gamma}$,

where $\gamma$ is any constant. To meet the wormhole geometry, it can be seen that $b^{\prime}\left(r_{0}\right)<1$ implies that $b^{\prime}\left(r_{0}\right)=\gamma<1$ and $b\left(r_{0}\right)=r_{0}$ hold. The asymptotically flat spacetime is also obtained for this shape function, i.e., $\frac{b(r)}{r}=r_{0}^{1-\gamma} r^{\gamma-1} \rightarrow 0$ as $r \rightarrow \infty$. Substituting the values of $\rho_{\mathrm{NCL}}$ and $b(r)$ from Eqs. (22) and (34) in Eq. (18), we obtain the following differential equation:

$$
\begin{aligned}
\frac{1}{r}[ & {\left[-\left(\frac{r}{r_{0}}\right)^{\gamma-1}-\sqrt{1-\left(\frac{r}{r_{0}}\right)^{\gamma-1}}\right] \frac{f^{\prime \prime}}{T^{\prime}} } \\
& +\frac{\gamma}{r^{2}}\left(\frac{r}{r_{0}}\right)^{\gamma-1} \frac{f^{\prime}}{T^{\prime}}-\frac{1}{r} \\
& \times\left[1-\left(\frac{r}{r_{0}}\right)^{\gamma-1}-\sqrt{1-\left(\frac{r}{r_{0}}\right)^{\gamma-1}}\right] \frac{T^{\prime \prime} f^{\prime}}{T^{\prime 2}} \\
= & \frac{M \sqrt{\phi}}{\pi^{2}\left(r^{2}+\phi\right)^{2}},
\end{aligned}
$$

where

$$
\begin{aligned}
T= & \frac{2}{r^{2}}\left[2-\left(\frac{r}{r_{0}}\right)^{\gamma-1}-2 \sqrt{1-\left(\frac{r}{r_{0}}\right)^{\gamma-1}}\right], \\
T^{\prime}= & -\frac{2}{r^{3}}\left[4 \left(1-\sqrt{\left.1-\left(\frac{r}{r_{0}}\right)^{\gamma-1}\right)-2\left(\frac{r}{r_{0}}\right)^{\gamma-1}}\right.\right. \\
& \left.+(\gamma-1)\left(\frac{r}{r_{0}}\right)^{\gamma-1}\left(1-\frac{1}{\sqrt{1-\left(\frac{r}{r_{0}}\right)^{\gamma-1}}}\right)\right],
\end{aligned}
$$


(i)

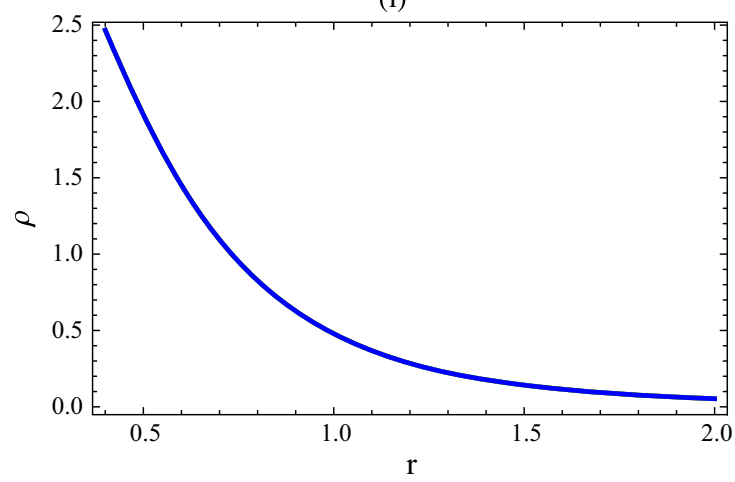

(ii)

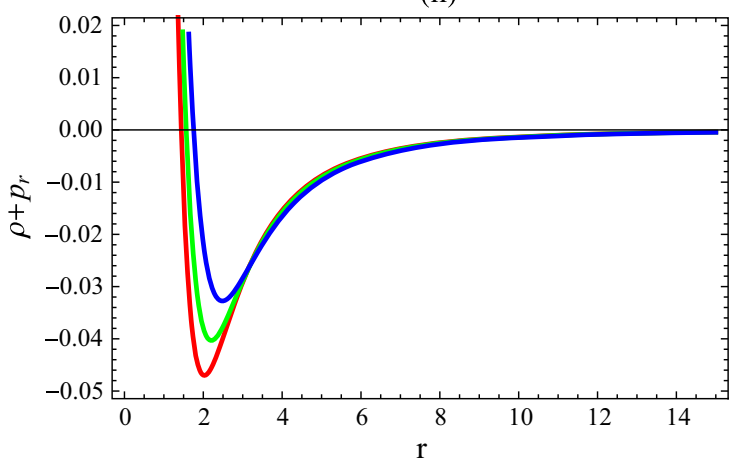

(iii)

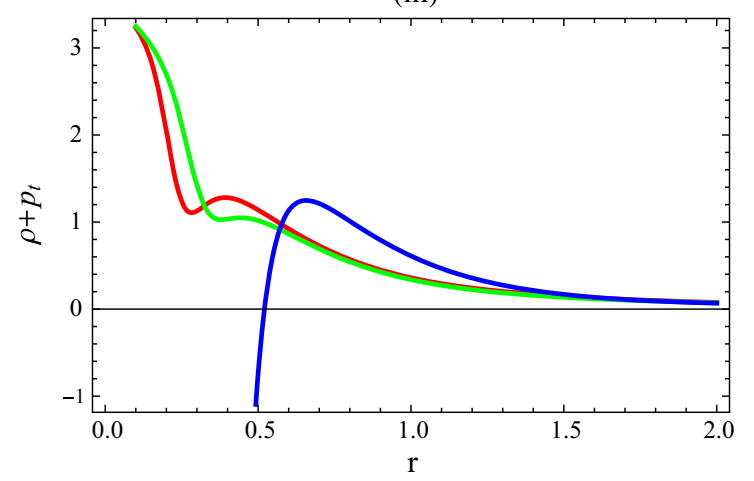

Fig. 4 Plots of WEC in $f(T)$ case: red curve for $\alpha=-2$, green curve for $\alpha=-3$, blue curve for $\alpha=-5$. Evolution of $\mathbf{i} \rho$ versus $r$, ii $\rho+p_{r}$ versus $r$, iii $\rho+p_{t}$ versus $r$

$$
\begin{aligned}
T^{\prime \prime}= & \frac{12}{r^{4}}\left[2\left(1-\sqrt{1-\left(\frac{r}{r_{0}}\right)^{\gamma-1}}\right)-\left(\frac{r}{r_{0}}\right)^{\gamma-1}\right. \\
& -\frac{(\gamma-1)(\gamma-6)}{6}\left(\frac{r}{r_{0}}\right)^{\gamma-1}\left(1-\frac{1}{\sqrt{1-\left(\frac{r}{r_{0}}\right)^{\gamma-1}}}\right) \\
& \left.+\frac{(\gamma-1)^{2}}{12}\left(\frac{r}{r_{0}}\right)^{2(\gamma-1)}\left(1-\left(\frac{r}{r_{0}}\right)^{\gamma-1}\right)^{-\frac{3}{2}}\right] .
\end{aligned}
$$

We evaluate the $f(T)$ function numerically and draw its plot as well as WEC versus $T$ and $r$, respectively, as shown in Fig. 5. Keeping the same values of NCL parameters along with $\gamma=0.5$ for three values of the throat radius, $r_{0}=0.93,0.95,0.99$. The plot i indicates the positively decreasing behavior of $f$.

The expressions for the WEC become

$$
\begin{aligned}
& \rho=\frac{\gamma}{r^{2}}\left(\frac{r}{r_{0}}\right)^{\gamma-1} \frac{f^{\prime}}{T^{\prime}}+\frac{1}{r}\left(1-\left(\frac{r}{r_{0}}\right)^{\gamma-1}\right.
\end{aligned}
$$

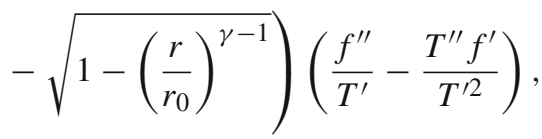

$$
\begin{aligned}
& \rho+p_{r}=\frac{\gamma-1}{r^{2}}\left(\frac{r}{r_{0}}\right)^{\gamma-1} \frac{f^{\prime}}{T^{\prime}}+\frac{1}{r}\left(1-\left(\frac{r}{r_{0}}\right)^{\gamma-1}\right. \\
& \left.-\sqrt{1-\left(\frac{r}{r_{0}}\right)^{\gamma-1}}\right)\left(\frac{f^{\prime \prime}}{T^{\prime}}-\frac{T^{\prime \prime} f^{\prime}}{T^{\prime 2}}\right), \\
& \rho+p_{t}=\frac{\gamma+1}{2 r^{2}}\left(\frac{r}{r_{0}}\right)^{\gamma-1} \frac{f^{\prime}}{T^{\prime}}+\frac{1}{2 r}\left(1-\left(\frac{r}{r_{0}}\right)^{\gamma-1}\right. \\
& \left.-\sqrt{1-\left(\frac{r}{r_{0}}\right)^{\gamma-1}}\right)\left(\frac{f^{\prime \prime}}{T^{\prime}}-\frac{T^{\prime \prime} f^{\prime}}{T^{\prime 2}}\right) .
\end{aligned}
$$

Figure 5(ii-iv) shows the plots of these expressions versus $r$. This shows that $\rho, \rho+p_{r}, \rho+p_{t}$ indicate a positive behavior of these expressions. Thus, physically acceptable wormhole solutions are obtained for the specific shape function where the basic role is played by the effective energy-momentum tensor.

\section{Equilibrium conditions}

To discuss the equilibrium configuration of the wormhole solutions, we consider the generalized Tolman-Oppenheimer -Volkov equation [48,49], 
(i)

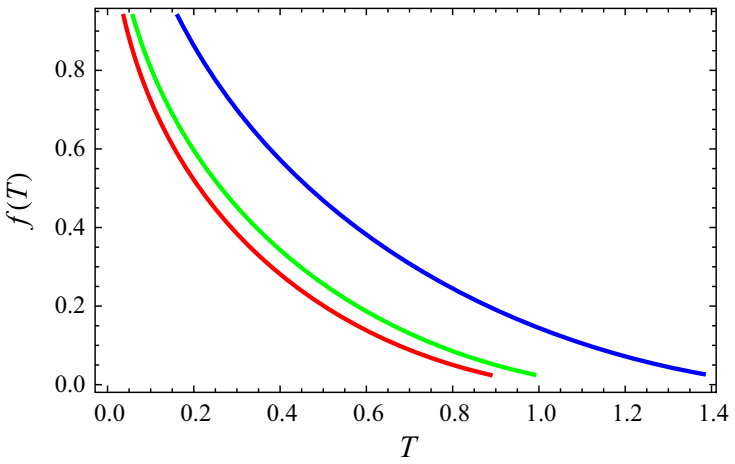

(iii)

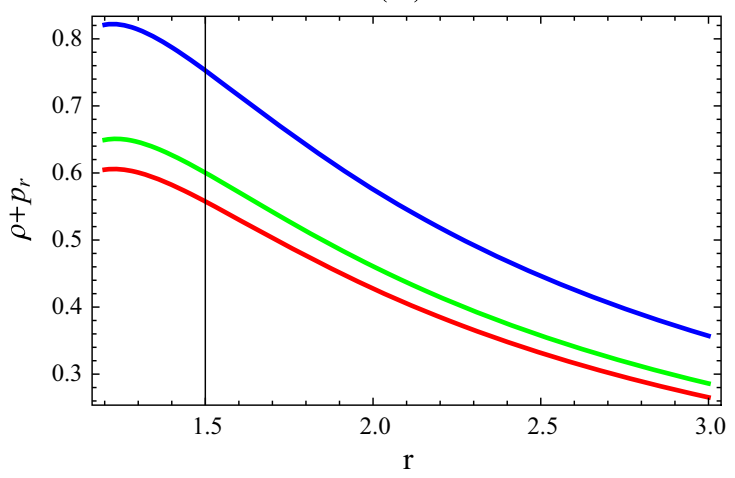

(ii)
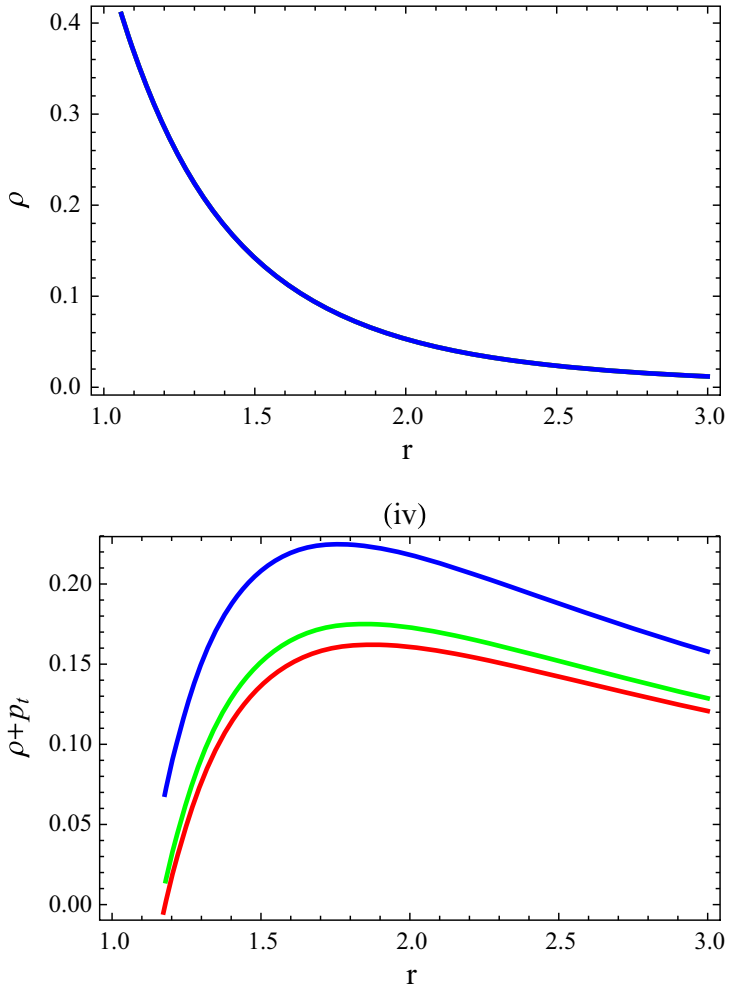

Fig. 5 Plots of $f(T)$ and WEC: red curve for $r_{0}=0.93$, green curve for $r_{0}=0.95$, blue curve for $r_{0}=0.99$. Evolution of $\mathbf{i} f(T)$ versus $T$, ii $\rho$ versus $r$, iii $\rho+p_{r}$ versus $r$, iv $\rho+p_{t}$ versus $r$

$\frac{\mathrm{d} p_{r}}{\mathrm{~d} r}+\frac{\sigma^{\prime}}{2}\left(\rho+p_{r}\right)+\frac{2}{r}\left(p_{r}-p_{t}\right)=0$,

for the metric $\mathrm{d} s^{2}=\operatorname{diag}\left(e^{\sigma(r)},-e^{\mu(r)},-r^{2},-r^{2} \sin ^{2} \theta\right)$. Ponce de León suggested this equation for an anisotropic mass distribution as follows:

$\frac{2}{r}\left(p_{t}-p_{r}\right)-\frac{e^{\frac{\mu-\sigma}{2}} \mathcal{M}_{\mathrm{eff}}}{r^{2}}\left(\rho+p_{r}\right)-\frac{\mathrm{d} p_{r}}{\mathrm{~d} r}=0$.

Here $\mathcal{M}_{\text {eff }}=\frac{1}{2} r^{2} e^{\frac{\sigma-\mu}{2}} \sigma^{\prime}$ is the effective gravitational mass which is measured from the throat to some arbitrary radius $r$. This equation indicates the equilibrium configuration for the wormhole solutions by taking gravitational, hydrostatic as well as anisotropic force due to an anisotropic matter distribution. Using Eq. (40), these forces are defined, respectively, as

$\mathcal{F}_{g f}=-\frac{\sigma^{\prime}\left(\rho+p_{r}\right)}{2}, \quad \mathcal{F}_{h f}=-\frac{\mathrm{d} p_{r}}{\mathrm{~d} r}, \quad \mathcal{F}_{a f}=\frac{2\left(p_{t}-p_{r}\right)}{r}$.

For the wormhole solutions to be in equilibrium, it is required that

$\mathcal{F}_{g f}+\mathcal{F}_{h f}+\mathcal{F}_{a f}=0$.

It is noted here that $\sigma$ represents the gravitational redshift, which is taken constant, i.e., $\sigma=2 \Psi$ leads to $\sigma^{\prime}=0$ for constant $\Psi$. This shows that $\mathcal{F}_{g f}$ becomes zero and we are left with hydrostatic and anisotropic forces with the corresponding equilibrium condition $\mathcal{F}_{h f}+\mathcal{F}_{a f}=0$. For the teleparallel case, specifically the $f(T)$ and $b(r)$ cases, $\mathcal{F}_{h f}$ and $\mathcal{F}_{a f}$ take the following form, respectively:

$$
\begin{aligned}
\mathcal{F}_{h f}= & -\frac{3}{r^{4}}\left[\frac{M}{2 \pi^{2}}\left(\frac{r \sqrt{\phi}}{r^{2}+\phi}+\tan ^{-1}\left(\frac{r}{\sqrt{\phi}}\right)\right)+c\right] \\
& +\frac{1}{r^{3}}\left[\frac{M \sqrt{\phi}\left(5 r^{3}+3 \phi\right)}{2 \pi^{2} r^{3}\left(r^{2}+\phi\right)^{2}} \frac{3 c}{r^{4}}-\frac{3 M}{2 \pi r^{4}} \tan ^{-1}\left(\frac{r}{\sqrt{\phi}}\right)\right], \\
\mathcal{F}_{a f}= & -\frac{1}{r^{3}}\left[\frac{M \sqrt{\phi}}{\pi^{2}\left(r^{2}+\phi\right)^{2}}-\frac{3}{r}\left\{\frac { M } { 2 \pi ^ { 2 } } \left(\frac{r \sqrt{\phi}}{r^{2}+\phi}\right.\right.\right. \\
& \left.\left.\left.+\tan ^{-1}\left(\frac{r}{\sqrt{\phi}}\right)\right)+c\right\}\right], \\
\mathcal{F}_{h f}= & \left.\frac{b^{\prime}}{r^{3}}-\frac{3 b}{r^{4}}\right)\left\{1+\frac{4 \alpha}{r^{2}}\left(2-\frac{b}{r}-2 \sqrt{1-\frac{b}{r}}\right)\right\} \\
& +\frac{b}{r^{6}}\left[-8 \alpha\left(2-\frac{b}{r}-2 \sqrt{1-\frac{b}{r}}\right)\right. \\
& \left.-\frac{4 \alpha\left(b^{\prime} r-b\right)}{r}\left(1-\frac{1}{\sqrt{1-\frac{b}{r}}}\right)\right]
\end{aligned}
$$




$$
\begin{aligned}
\mathcal{F}_{a f}= & -\frac{1}{r^{3}}\left[( b ^ { \prime } - \frac { 3 b } { r } ) \left\{1+\frac{4 \alpha}{r^{2}}(2\right.\right. \\
& \left.\left.-\frac{b}{r}-2 \sqrt{1-\frac{b}{r}}\right)\right\}+\frac{4 \alpha}{r}\left(\frac{b}{r}-1\right. \\
& \left.+\sqrt{1-\frac{b}{r}}\right)\left[4\left(1-\sqrt{1-\frac{b}{r}}\right)-\frac{2 b}{r}\right. \\
& \left.\left.+\frac{b^{\prime} r-b}{r}\left(1-\frac{1}{\sqrt{1-\frac{b}{r}}}\right)\right]\right], \\
\mathcal{F}_{h f}= & \frac{\gamma-3}{r^{3}}\left(\frac{r}{r_{0}}\right)^{\gamma-1} \frac{f^{\prime}}{T^{\prime}}+\frac{1}{r^{2}}\left(\frac{r}{r_{0}}\right)^{\gamma-1}\left(\frac{f^{\prime \prime}}{T^{\prime}}-\frac{T^{\prime \prime} f^{\prime}}{T^{\prime 2}}\right), \\
\mathcal{F}_{a f}= & \frac{3-\gamma}{r^{3}}\left(\frac{r}{r_{0}}\right)^{\gamma-1} \frac{f^{\prime}}{T^{\prime}}+\frac{1}{r^{2}}\left(\left(\frac{r}{r_{0}}\right)^{\gamma-1}\right. \\
& \left.-1+\sqrt{1-\left(\frac{r}{r_{0}}\right)^{\gamma-1}}\right)\left(\frac{f^{\prime \prime}}{T^{\prime}}-\frac{T^{\prime \prime} f^{\prime}}{T^{\prime 2}}\right) .
\end{aligned}
$$

We plot these equations for the obtained wormhole solutions as shown in Figs. 6, 7, and 8, respectively. For the teleparallel case, we examine that hydrostatic and anisotropic forces show the same behavior but in opposite directions and thus balance each other. This implies that the wormhole solution in the teleparallel case satisfies the equilibrium condition. In the case of a power-law $f(T)$ function, this condition shows wormhole solutions in an equilibrium state as $r$ increases. For smaller values of $r$, these solutions do not satisfy the equilibrium condition properly or we may remark that these solutions are less stable. For a specific $b(r)$ function, the forces $\mathcal{F}_{h f}$ and $\mathcal{F}_{a f}$ do not balance each other. Since all the curves behave in opposite manners there is no similar-

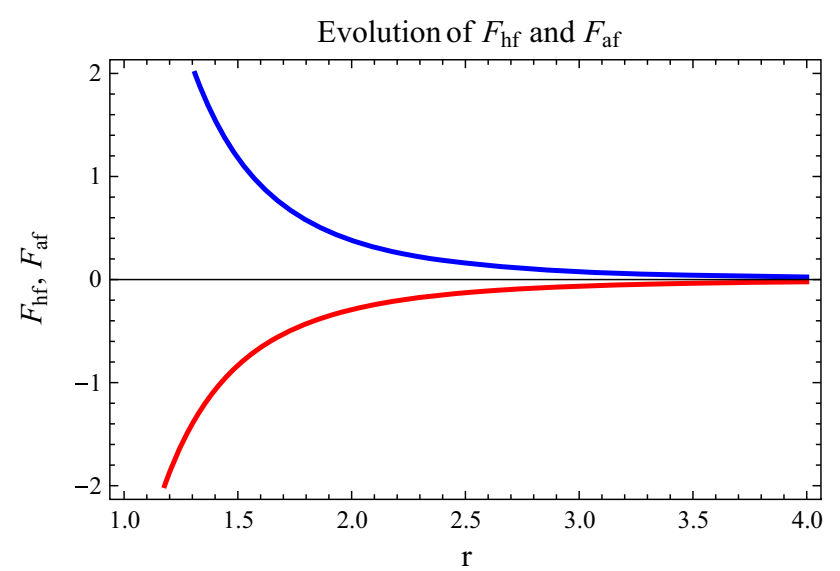

Fig. 6 Plot of $\mathcal{F}_{h f}$ and $\mathcal{F}_{a f}$ in teleparallel case versus $r$ : red curve represents $\mathcal{F}_{h f}$ and blue curve represents $\mathcal{F}_{\text {af }}$. Evolution of $\mathcal{F}_{h f}$ and $\mathcal{F}_{\text {af }}$

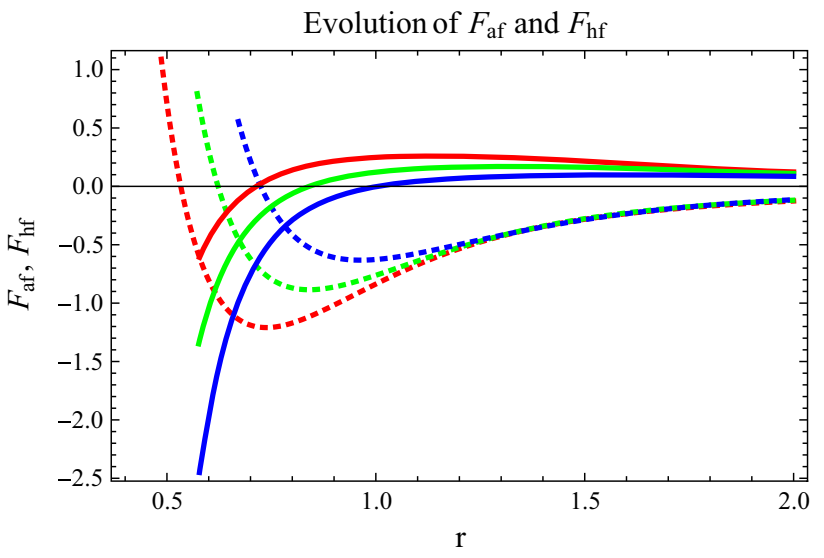

Fig. 7 Plot of $\mathcal{F}_{h f}($ dotted $)$ and $\mathcal{F}_{\text {af }}$ (solid) in specific $f(T)$ case versus $r$. Evolution of $\mathcal{F}_{a f}$ and $\mathcal{F}_{h f}$

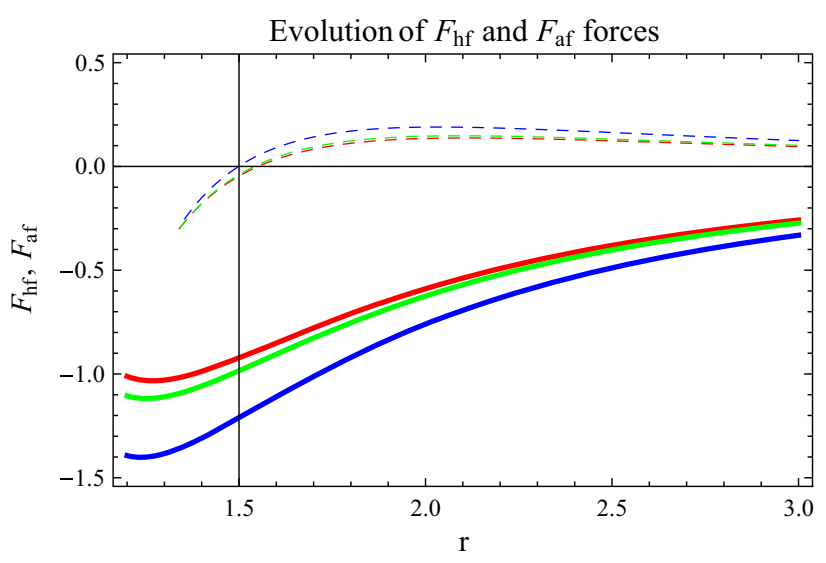

Fig. 8 Plot of $\mathcal{F}_{h f}($ dotted $)$ and $\mathcal{F}_{\text {af }}$ (solid) in specific $b(r)$ case versus $r$. Evolution of $\mathcal{F}_{h f}$ and $\mathcal{F}_{a f}$ forces

ity. Therefore the physically acceptable wormhole solutions are not in equilibrium form.

\section{Conclusion}

A wormhole represents a shortcut distance to connect different regions of the universe. To study these solutions, the violation of NEC plays the key role which is associated with the exotic matter. To minimize the usage of exotic matter is an important issue, which leads one to explore a realistic model in favor of the wormhole. In this paper, we have studied static spherically symmetric wormhole solutions in $f(T)$ gravity by taking a NCL background. We have developed the $f(T)$ field equations in terms of an effective energy-momentum tensor by taking a non-diagonal tetrad and proved that this tensor is responsible for the WEC violation. By imposing the condition on the matter content to thread the wormhole solutions, we have assumed either the $f(T)$ or the shape function and constructed the other one. The graphical behavior of these solutions is discussed. 
For the power-law $f(T)$ model, we have discussed two cases: teleparallel gravity and $f(T)$ gravity in quadratic form. Both models satisfied the conditions for the wormhole geometry as regards the shape function. The WEC condition is violated for the first case, while the possibility of a tiny wormhole solution is examined for the $f(T)$ case. In another case of particular form of power-law shape function, we have analyzed the wormhole geometry. We have found physically acceptable wormhole solutions as the WEC is satisfied in this case. Also, we have examined the stability of these wormhole solutions with the help of a generalized Tolman-OppenheimerVolkov equation. We have found that teleparallel NCL wormhole solutions are stable, while $f(T)$ NCL wormhole solutions are less stable. For the wormhole solutions in a specific shape function case, we have obtained unstable solutions.

Bhar and Rahaman [35] investigated whether the wormhole solutions exist in different dimensional noncommutative inspired spacetimes with a Lorentzian distribution. For four- and five-dimensional spacetime, there exist wormhole solutions, while no solution exists for higher dimensions. They observed a stable wormhole, i.e., satisfying the flare out conditions and violating the energy conditions for four dimensions. It is interesting to note that for the underlying case, we also obtain asymptotically flat and stable solutions in the teleparallel case, i.e., the behavior of all conditions is identical. The wormhole solutions [32] in the background of a NC geometry give physically acceptable wormhole solutions in the $f(T)$ gravity case, while in the teleparallel case, the energy conditions are violated. However, this work is done taking a diagonal tetrad, which is less of interest for spherical symmetry. In the case of a Lorentzian distributed NC background, we have found physically acceptable wormhole solutions in a more stable form. We have used a non-diagonal tetrad, which is the favorable choice for spherical symmetry. The NC wormholes in $f(R)$ gravity with a Lorentzian distribution are discussed by Rahaman et al. [52]. They studied the same cases and found a violation of the energy conditions in all cases. That is, there are no physically acceptable solutions in $f(R)$ gravity. However, we have found physically acceptable as well as stable solutions.

Open Access This article is distributed under the terms of the Creative Commons Attribution 4.0 International License (http://creativecomm ons.org/licenses/by/4.0/), which permits unrestricted use, distribution, and reproduction in any medium, provided you give appropriate credit to the original author(s) and the source, provide a link to the Creative Commons license, and indicate if changes were made. Funded by SCOAP ${ }^{3}$.

\section{References}

1. A.Y. Kamenshchik, U. Moschella, V. Pasquier, Phys. Lett. B 511, $265(2001)$
2. M. Li, Phys. Lett. B 603, 1 (2004)

3. R.G. Cai, Phys. Lett. B 657, 228 (2007)

4. H. Wei, Commun. Theor. Phys. 52, 743 (2009)

5. A. Sheykhi, M. Jamil, Gen. Relativ. Gravit. 43, 2661 (2011)

6. R. Ferraro, F. Fiorini, Phys. Rev. D 75, 084031 (2007)

7. R. Ferraro, F. Fiorini, ibid. 78, 124019 (2008)

8. T. Wang, Phys. Rev. D 84, 024042 (2011)

9. T. Wang, JCAP 11, 033 (2011)

10. M.H. Daouda, M.E. Rodrigues, M.J.S. Houndjo, Eur. Phys. J. C. 71, 1817 (2011)

11. R. Ferraro, F. Fiorini, Phys. Rev. D 84, 083518 (2011)

12. M.H. Daouda, M.E. Rodrigues, M.J.S. Houndjo, Eur. Phys. J. C 72, $1890(2012)$

13. P.A. Gonzlez, E.N. Saridakis, Y. Vsquez, JHEP 53, 2012 (2012)

14. G.G.L. Nashed, Chin. Phys. Lett. 29, 050402 (2012)

15. L. Iorio, E.N. Saridakis, Mon. Not. R. Astron. Soc. 427, 1555 (2012)

16. S. Capozziello et al., JHEP 1302, 039 (2013)

17. K. Atazadeh, M. Mousavi, Eur. Phys. J. C 73, 2272 (2013)

18. G.G.L. Nashed, Phys. Rev. D 88, 104034 (2013)

19. A. Paliathanasis, Phys. Rev. D 89, 104042 (2014)

20. J. Aftergood, A. DeBenedictis, Phys. Rev. D 90, 124006 (2014)

21. G. Kofinas, E. Papantonopoulos, E.N. Saridakis, arXiv: 1501.00365

22. G.G.L. Nashed, Gen. Relativ. Gravit. 45, 1887 (2013)

23. S.-W. Kim, H. Lee, Phys. Rev. D 63, 064014 (2001)

24. F. Rahaman, S. Islam, P.K.F. Kuhfittig, S. Ray, Phys. Rev. D 86, 106010 (2012)

25. M. Sharif, A. Jawad, Eur. Phys. J. Plus 129, 15 (2014)

26. F. Rahaman et al., Int. J. Theor. Phys. 53, 1910 (2014)

27. C.G. Böhmer, T. Harko, F.S.N. Lobo, Phys. Rev. D 85, 044033 (2012)

28. M. Jamil, D. Momeni, R. Myrzakulov, Eur. Phys. J. C 73, 2267 (2013)

29. M. Sharif, S. Rani, Gen. Relativ. Gravit. 45, 2389 (2013)

30. F. Rahaman, S. Islam, P.K.F. Kuhfittig, S. Ray, Phys. Rev. D 86, 106010 (2012)

31. M. Jamil et al., J. Korean Phys. Soc. 65, 97 (2014)

32. M. Sharif, S. Rani, Phys. Rev. D 88, 123501 (2013)

33. M. Sharif, S. Rani, Eur. Phys. J. Plus 129, 237 (2014)

34. M. Sharif, S. Rani, Adv. High Energy Phys. 2014, 691497 (2014)

35. P. Bhar, F. Rahaman, Eur. Phys. J. C 74, 3213 (2014)

36. C.W. Misner, J.A. Wheeler, Ann. Phys. 2, 525 (1957)

37. J.A. Wheeler, Geometrodynamics (Acedamic Press, New York, 1962)

38. M. Morris, K. Thorne, Am. J. Phys. 56, 395 (1988)

39. M. Visser, S. Kar, N. Dadhich, Phys. Rev. Lett. 90, 201102 (2003)

40. V.C. De Andrade, J.G. Pereira, Gen. Relat. Gravit. 30, 263 (1998)

41. R. Aldrovandi, J.G. Pereira, Teleparallel Gravity: An Introduction (Springer, New York, 2012)

42. B. Li, T.P. Sotiriou, J.D. Barrow, Phys. Rev. D 83, 064035 (2011)

43. B. Li, T.P. Sotiriou, J.D. Barrow, ibid. 83, 104017 (2011)

44. T.P. Sotiriou, B. Li, J.D. Barrow, Phys. Rev. D 83, 104030 (2011)

45. D. Liu, M.J. Rebouças, Phys. Rev. D 86, 083515 (2012)

46. C.G. Böhmer, A. Mussa, N. Tamanini, Class. Quantum Gravity 28 , 245020 (2011)

47. P. Nicolini, A. Smailagic, E. Spalluci, Phys. Lett. B 632, 547 (2006)

48. F. Rahaman, P.K.F. Kuhfittig, S. Ray, N. Islamd, Eur. Phys. J. C 74, 2750 (2014)

49. P.K.F. Kuhfittig, Eur. Phys. J. C 74, 2818 (2014)

50. M. Jamil, M.U. Farooq, Int. J. Theor. Phys. 49, 835 (2010)

51. M. Farooq, M. Akbar, M. Jamil, AIP Conf. Proc. 1295, 176 (2010)

52. F. Rahaman, A. Banerjee, M. Jamil, A.K. Yadav, H. Idris, Int. J. Theor. Phys. 53, 1910 (2014) 